\title{
共挤出法制备双层中空纤维陶瓷复合膜
}

\author{
刘金云，张玉亭，洪 周，刘 华，王圣贤，顾学红 \\ (南京工业大学 化工学院, 材料化学工程国家重点实验室, 南京 210009)
}

摘 要: 中空纤维陶瓷膜具有装填密度高, 传质阻力低, 使用寿命长等优点, 被广泛用于膜分离领域。高度非对称结 构的中空纤维膜有利于同时实现高通量与高截留率, 本研究采用共挤出法制备双层中空纤维陶瓷复合膜, 内外层 纺丝液分别掺杂平均粒径为 $1 \mu \mathrm{m}$ 和 $300 \mathrm{~nm}$ 的 $\alpha-\mathrm{Al}_{2} \mathrm{O}_{3}$ 粉体。系统考察了内层纺丝液 $\mathrm{TiO}_{2}$ 掺杂量、外层纺丝液 $\mathrm{Al}_{2} \mathrm{O}_{3}$ / 聚醚砜(PESf)质量比和瑖烧温度对膜的结构与性能的影响。结果表明, 在内层纺丝液 $\mathrm{TiO}_{2}$ 掺杂量为 $2 \mathrm{wt} \%$, 外 层纺丝液 $\mathrm{Al}_{2} \mathrm{O}_{3} / \mathrm{PESf}$ 质量比为 5.60 , 烧结温度为 $1350{ }^{\circ} \mathrm{C}$ 的最优条件下, 中空纤维膜断裂负荷为 $24 \mathrm{~N}$ 、平均孔径为 $0.15 \mu \mathrm{m}$ 、去油率为 $97.5 \%$ 。

关 键 词: 双层中空纤维; 共挤出法; $\mathrm{TiO}_{2} ; \mathrm{Al}_{2} \mathrm{O}_{3} / \mathrm{PESf}$

中图分类号: TQ174 文献标识码: A

\section{Fabrication of Dual-layer Hollow Fiber Ceramic Composite Membranes by Co-extrusion}

\author{
LIU Jinyun, ZHANG Yuting, HONG Zhou, LIU Hua, WANG Shengxian, GU Xuehong
}

(State Key Laboratory of Materials-Oriented Chemical Engineering, College of Chemistry and Chemical Engineering, Nanjing Tech University, Nanjing 210009, China)

\begin{abstract}
Hollow fiber ceramic membranes have been widely accepted in membrane separation due to their advantages of high packing density, low transfer resistance and long-period operation. Fabrication of highly-asymmetric hollow fiber membrane is helpful to achieve high flux as well as high rejection simultaneously. In this work, dual-layer hollow fiber ceramic composite membranes were prepared by the co-extrusion method. The inner and outer suspensions were doped with $\alpha-\mathrm{Al}_{2} \mathrm{O}_{3}$ powders with average particle sizes of $1 \mu \mathrm{m}$ and $300 \mathrm{~nm}$ respectively. Effects of $\mathrm{TiO}_{2}$ content in inner suspension, $\mathrm{Al}_{2} \mathrm{O}_{3}$ /polyether sulfone (PESf) mass ratio of outer suspension and calcination temperature on structure and properties of membrane were investigated extensively. When the $\mathrm{TiO}_{2}$ content was $2 \mathrm{wt} \%$ in inner suspension, the $\mathrm{Al}_{2} \mathrm{O}_{3} / \mathrm{PESf}$ mass ratio was 5.60 in outer suspension and the sintering temperature was $1350{ }^{\circ} \mathrm{C}$, the hollow fiber membrane got the optimum performance, with fracture load of $24 \mathrm{~N}$, average pore size of $0.15 \mu \mathrm{m}$, and oil rejection of $97.5 \%$.
\end{abstract}

Key words: dual-layer hollow fiber; co-extrusion; $\mathrm{TiO}_{2} ; \mathrm{Al}_{2} \mathrm{O}_{3} / \mathrm{PESf}$

中空纤维膜在腐蚀性流体过滤、高温高压膜反 应器、固体燃料电池等领域受到广泛关注 ${ }^{[1-4]}$, 主要

收稿日期: 2020-04-07; 收到修改稿日期：2020-06-12

基金项目: 国家自然科学基金面上项目(21776128); 江苏省“333 高层次人才培养工程”资助项目(第二层次); 江苏省自然科 学基金青年项目(BK20170132)

National Natural Science Foundation of China (21776128); “333 Talent project” of Jiangsu Province; Natural Science Foundation of Jiangsu Province (BK20170132)

作者简介: 刘金云(1994-), 女, 硕士研究生. E-mail: 1158381254@qq.com LIU Jinyun(1994-), female, Master candidate. E-mail: 1158381254@qq.com

通讯作者: 顾学红, 教授. E-mail: Xhgu@njtech.edu.cn GU Xuehong, professor. E-mail: Xhgu@njtech.edu.cn 
采用相转化法制备, 由于非对称结构和多孔结构 使其具有低传输阻力和高通量的特点。另外它还具 有装填密度高、化学稳定性好以及耐溶剂性等突出 优势 ${ }^{[5-7]}$ 。

普通单层中空纤维膜往往难以兼具高通量和高 截留率, 需要二次涂覆改善膜的分离性能。双层膜 可以简化二次涂覆过程, 节省膜制备时间, 因而受 到研究者的关注。Henne 等 $^{[8]}$ 发明了共挤出法并制 备了双层中空纤维复合膜用于临床血液透析。许多 研究者围绕双层聚合物中空纤维膜在膜制备方法、 膜形貌的控制以及双层之间黏附和分层的机理等方 面开展了大量研究 ${ }^{[9-12]}$, 并将膜成功用于气体分离、 渗透汽化、膜蒸馏等领域 ${ }^{[13-15]}$ 。21 世纪以来, 双层 无机中空纤维膜逐渐受到研究者的关注。2004 年, Jong 等 ${ }^{[16]}$ 首次制备了双层氧化铝中空纤维膜, 虽然 膜的强度较低, 但内外两层结合得很好, 且无分层 现象。之后, Droushiotis ${ }^{[17]}$ 和 Othman ${ }^{[18]}$ 等在研究微 管式固体氧化物燃料电池(MT-SOFC)时, 采用共挤 出和共烧结法制备了电解液/阳极双层中空纤维，与 传统耗时的多步法相比，该方法不仅缩短了制备时 间，降低了制备成本，而且可以调控外层厚度，优 化其本身性能 ${ }^{[19-20]}$ 。此外, 双层陶瓷中空纤维在膜

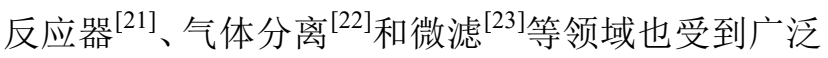
关注。

本课题组前期开发出四通道陶瓷中空纤维膜, 系统研究了中空纤维膜的结构与性能之间的构效关 系 ${ }^{[24-25]}$ 。与普通单通道陶瓷中空纤维膜相比, 四通 道陶瓷中空纤维膜的机械强度显著提升。当调节膜 的结构缩小其孔径时, 膜的通量大幅下降, 影响了 膜的传质效率。本研究采用共挤出法和共烧结法制 备四通道双层中空纤维复合陶瓷膜, 同时获得了较 小的孔径和较高的通量, 系统考察了内层助烧剂的 掺杂量、外层 $\mathrm{Al}_{2} \mathrm{O}_{3} / \mathrm{PESf}$ 质量比和烧结温度对膜的 结构和性能的影响。

\section{1 实验方法}

\section{1 实验原料}

纺丝液陶瓷粉体选用 $\alpha-\mathrm{Al}_{2} \mathrm{O}_{3}(1 \mu \mathrm{m}$ 和 $300 \mathrm{~nm}$, 国内工业级)和 $\mathrm{TiO}_{2}(1 \mu \mathrm{m}$, 锐钛矿, 国内工业级)。 溶剂为 $N$-甲基-2-吡咯烷酮(NMP, 纯度 99\%, 国药 集团化学试剂有限公司), 粘合剂为聚醚砜(PESf, BD-5, 国内工业级), 添加剂为聚乙烯吡咯烷酮 K30 (PVP, 国药集团化学试剂有限公司), 内外凝固浴用 去离子水。

\section{2 双层中空纤维陶瓷膜的制备}

采用相转化与高温煆烧相结合的方法制备中空 纤维陶瓷膜: 首先 NMP、PESf 和 PVP 以一定比例 充分搅拌均匀, 形成聚合物溶液; 然后加入陶瓷粉 体, 继续搅拌 $24 \mathrm{~h}$ 形成纺丝液, 纺丝液的组成比例 如表 1 所示。纺丝液在注入不锈钢注射器之前, 先 抽真空 $2 \mathrm{~h}$, 脱去铸膜液里的气泡。然后内外层纺丝 液在注射洜的推动下分别以 35 和 $8 \mathrm{~mL} / \mathrm{min}$ 的速 度穿过四通道双层纺丝头, 并用去离子水作为内凝 固浴在压力推动下以 $40 \mathrm{~mL} / \mathrm{min}$ 进入, 空气间距为 $10 \mathrm{~cm}$ 。纺丝液挤出成形后在外凝固浴中静置 $24 \mathrm{~h}$ 后取出晾干, 在空气气氛中对纺丝生坏进行高温㷽 烧, 首先以 $2{ }^{\circ} \mathrm{C} / \mathrm{min}$ 的升温速率升温到 $600{ }^{\circ} \mathrm{C}$, 然 后以 $1{ }^{\circ} \mathrm{C} / \mathrm{min}$ 的升温速率升到设定温度, 并保温 $5 \mathrm{~h}$, 最后以 $2{ }^{\circ} \mathrm{C} / \mathrm{min}$ 的速率降至室温。

\section{3 性能测试}

利用扫描电子显微镜(SEM, S4800, 日立)观察 中空纤维膜的表面和断面形貌, 利用 $\mathrm{X}$ 射线衍射仪 (XRD, MiniFlex 600, Rigaku)分析中空纤维膜的晶 型结构。采用电子万能试验机(CMI6203, 深圳市新 三思材料检测有限公司)测试双层中空纤维膜的三 点弯曲强度, 其中跨距为 $40 \mathrm{~mm}$, 十字头下降速度 为 $0.5 \mathrm{~mm} / \mathrm{min}$, 每根样品至少选取 5 根。采用微滤 膜孔径分析仪(PSDA-20, 南京高谦功能材料科技有 限公司)测试中空纤维膜的孔径, 通过气体泡压法 测定最大孔径、平均孔径及孔径分布。

在 $0.1 \mathrm{MPa}$ 压差作用下测试膜的纯水渗透通量 $(J)$, 计算公式如下:

$$
J=\frac{V}{A \cdot t}
$$

其中, $V$ 为纯水透过总量, $\mathrm{m}^{3} ; A$ 为有效表面积, $\mathrm{m}^{2} ; t$ 为过滤时间, $\mathrm{h}$ 。

通过阿基米德排水法测定陶瓷中空纤维膜的孔 隙率，计算公式如下:

$$
q=\frac{G_{2}-G_{1}}{G_{2}-G_{3}} \times 100 \%
$$

表 1 双层中空纤维膜纺丝液组成/wt $\%$

Table 1 Suspension composition of dual-layer hollow fiber membrane/wt\%

\begin{tabular}{ccc}
\hline Suspension composition & Inner layer & Outer layer \\
\hline Solvent $(\mathrm{NMP})$ & 33.34 & 37.81 \\
$\mathrm{Al}_{2} \mathrm{O}_{3}(1 \mu \mathrm{m})$ & 55.35 & $/$ \\
$\mathrm{Al}_{2} \mathrm{O}_{3}(300 \mathrm{~nm})$ & $/$ & 49.15 \\
Polymer $(\mathrm{PESf})$ & 9.77 & 11.34 \\
Additive (PVP) & 1.54 & 1.70 \\
\hline
\end{tabular}


其中, $q$ 为中空纤维陶瓷膜的孔隙率, $\% ; G_{1}$ 为样品 在空气中完全干燥的质量, $\mathrm{g} ; G_{2}$ 为吸水饱和样品在 空气中的质量, $\mathrm{g} ; G_{3}$ 为吸水饱和样品在水中的重 量, $g$ 。

通过膜烧结前后直径的变化计算膜的径向收缩 率 $\eta$, 公式如下:

$$
\eta=\frac{D_{\mathrm{P}}-D_{\mathrm{S}}}{D_{\mathrm{P}}} \times 100 \%
$$

其中, $D_{\mathrm{P}}$ 为生坏直径, $\mu \mathrm{m} ; D_{\mathrm{S}}$ 为烧结后直径, $\mu \mathrm{m}$ 。

实验室自制水包油乳液体系(豆油和去离子水), 油浓度为 $200 \mathrm{mg} / \mathrm{L}$, 油滴粒径分布在 0.06 10.00 $\mu \mathrm{m}$ 之间。通过自制错流膜分离装置进行油水分离, 使 用总有机碳分析仪(TOC, Shimadzu ASI-5000)检测 进料和渗透物中的 $\mathrm{TOC}$ 值。膜的去油率 $R_{\mathrm{TOC}}$ 计算 公式如下:

$$
R_{\mathrm{TOC}}=\frac{C_{\mathrm{F}}-C_{\mathrm{P}}}{C_{\mathrm{F}}} \times 100 \%
$$

其中, $C_{\mathrm{P}}$ 为进料液 $\mathrm{TOC}$ 值, $\mathrm{mg} / \mathrm{L} ; C_{\mathrm{F}}$ 为渗透液 $\mathrm{TOC}$ 值, $\mathrm{mg} / \mathrm{L}$ 。

\section{2 结果与讨论}

\section{1 单层与双层中空纤维陶瓷膜比较}

首先选用 $1 \mu \mathrm{m}$ 和 $300 \mathrm{~nm}$ 的 $\mathrm{Al}_{2} \mathrm{O}_{3}$ 粉体分别制 备两种单层中空纤维膜和 $1 \mu \mathrm{m} / 300 \mathrm{~nm}$ 双层中空纤 维膜, 膜的烧结温度为 $1350{ }^{\circ} \mathrm{C}$ 。如图 1 所示, 双层 中空纤维膜生坏的外径是 $4.67 \mathrm{~mm}$, 烧结后外径减 小为 $4 \mathrm{~mm}$, 径向收缩率为 $14.3 \%$ 。其中双层膜生坏 的外层厚度约为 $100 \mu \mathrm{m}$ (图 1(a,b)), 高温烧结后外 层厚度收缩至 $60 \mu \mathrm{m}$ (图 1(d))。与 $1 \mu \mathrm{m}$ 单层中空纤 维膜相比(图 1(e, f)), 双层中空纤维膜的外径更大,
断面具有明显的双层结构, 内外两层结合紧密，且 无明显分层现象。由表面形貌看出, 单层膜表面粗 䊁度较高, 孔径分布不均(图 1(h)), 双层膜则由于外 层 $\mathrm{Al}_{2} \mathrm{O}_{3}$ 颗粒较小, 膜表面更加光滑平整(图 $1(\mathrm{~g})$ ), 孔径也都小于 $0.24 \mu \mathrm{m}$, 适合用于微滤或者作为复 合膜分离层的载体。

表 2 为单层与双层中空纤维膜的性能对比, 如 表所示, 粉体粒径越小, 纤维膜的径向收缩率越大。 当粉体粒径为 $1 \mu \mathrm{m}$ 时, 膜的径向收缩率最小, 孔隙 率和平均孔径最大, 因此膜的纯水通量最高, 同时 油截留率最低。由于粉体间隙较大, 相互熔融烧结 程度较低, 因此膜的断裂负荷最低。与之相比, 双层 膜由于外层粉体粒径较小, 膜层较为致密, 膜的平 均孔径和纯水通量有所降低, 油截留率则提高至 97\%，同时膜的断裂负荷也有所增强。Chong 等 ${ }^{[23]}$ 在不锈钢双层中空纤维的外层掺杂了小颗粒的钇稳 定型氧化锆(YSZ)粉体，发现膜的孔径也明显减小。 双层中空纤维膜与 $300 \mathrm{~nm}$ 单层中空纤维相比, 油截 留率略有下降, 但是纯水通量显著提高了 1.6 倍。膜 的断裂负荷相比也较低, 表明双层中空纤维膜的机 械强度主要由内层决定, 进一步通过在内层纺丝液 中掺杂 $1 \mu \mathrm{m} \mathrm{TiO}_{2}$ 粉体作为烧结助剂来提高膜的机 械强度。

\section{2 双层膜内层 $\mathrm{TiO}_{2}$ 掺杂量的影响}

图 2 为不同 $\mathrm{TiO}_{2}$ 掺杂量下双层中空纤维膜的 XRD 图谱。如图所示, 掺杂 $\mathrm{TiO}_{2}$ 的双层中空纤维膜 产生了新的钛酸铝 $\left(\mathrm{Al}_{2} \mathrm{TiO}_{5}\right)$ 峰, 且峰强度随着 $\mathrm{TiO}_{2}$ 掺 杂量的增加逐渐变强, 同时 $\mathrm{Al}_{2} \mathrm{O}_{3}$ 峰的强度逐渐减 弱。这是因为 $\mathrm{TiO}_{2}$ (锐钛矿) 在升温 $\left(>600{ }^{\circ} \mathrm{C}\right.$ )过程中 首先转化成 $\mathrm{TiO}_{2}$ (金红石), 然后在高温下 $\left(>1280{ }^{\circ} \mathrm{C}\right)$ 与 $\mathrm{Al}_{2} \mathrm{O}_{3}$ 发生如下反应:
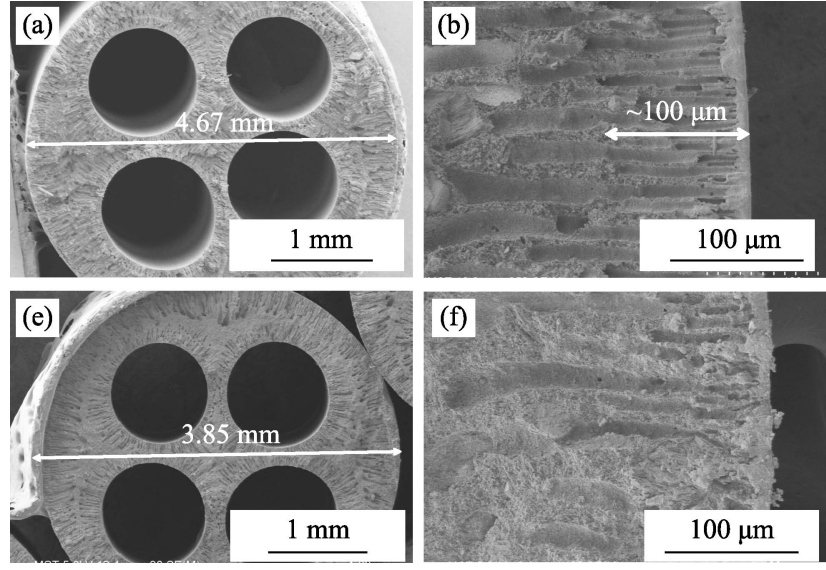
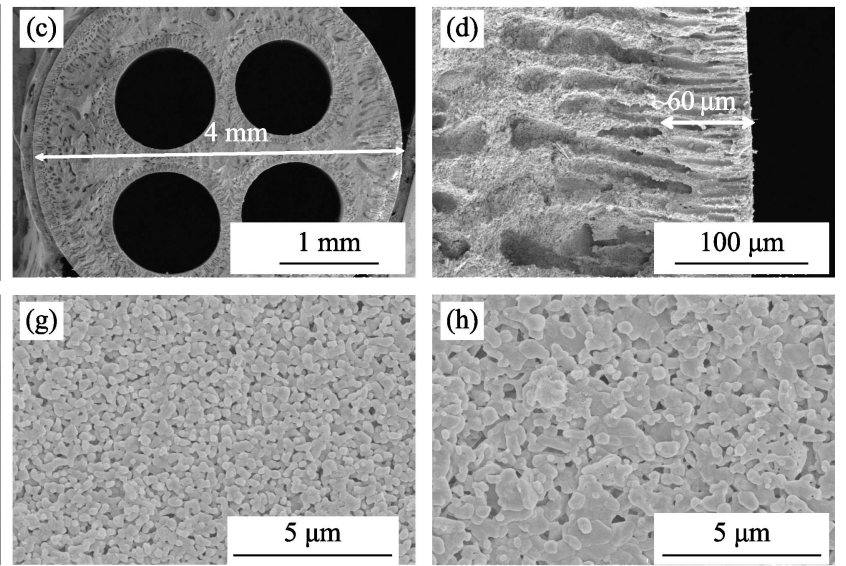

图 1 双层中空纤维膜和单层中空纤维膜 SEM 照片

Fig. 1 SEM images of dual-layer hollow fiber and single-layer hollow fiber

Cross-section morphologies of (a, b) dual-layer hollow fiber precursor, (c, d) dual-layer hollow fiber, (e, f) $1 \mu$ m single-layer hollow fiber; SEM images of the surface morphology: (g) dual-layer hollow fiber, (h) $1 \mu \mathrm{m}$ single-layer hollow fiber 
表 2 单层与双层中空纤维膜的性能比较

Table 2 Comparison of properties between single-layer and dual-layer hollow fiber membranes

\begin{tabular}{ccccccc}
\hline Property & $\eta / \%$ & Fracture load $/ \mathrm{N}$ & Pure water flux $/\left(\mathrm{m}^{3} \cdot \mathrm{m}^{-2} \cdot \mathrm{h}^{-1}\right)$ & Porosity $/ \%$ & Average pore size $/ \mu \mathrm{m}$ & $R_{\mathrm{TOC}} / \%$ \\
\hline Single-layer $(1 \mu \mathrm{m})$ & 13.0 & 9 & 3.52 & 51.57 & 0.70 & 95.4 \\
Single-layer $(300 \mathrm{~nm})$ & 16.9 & 19 & 0.86 & 48.20 & 0.13 & 98.4 \\
Dual-layer $(1 \mu \mathrm{m} / 300 \mathrm{~nm})$ & 14.3 & 12 & 2.30 & 50.89 & 0.24 & 97 \\
\hline
\end{tabular}

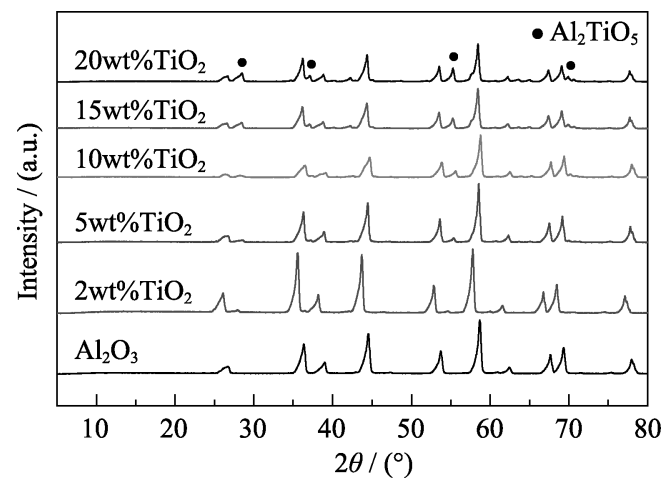

图 2 不同 $\mathrm{TiO}_{2}$ 掺杂量下双层中空纤维膜的 XRD 图谱

Fig. 2 XRD patterns of dual-layer hollow fiber membranes with different $\mathrm{TiO}_{2}$ contents

$$
\alpha-\mathrm{Al}_{2} \mathrm{O}_{3}+\mathrm{TiO}_{2} \text { (rutile) }=\beta-\mathrm{Al}_{2} \mathrm{TiO}_{5}
$$

在 $\mathrm{Al}_{2} \mathrm{TiO}_{5}$ 结构中, 每个 $\mathrm{Al}^{3+}$ 和 $\mathrm{Ti}^{4+}$ 都被六个 $\mathrm{O}^{2-}$ 包围, 形成扭曲的八面体, 属于板钛矿晶型, 这 些八面体形式的定向双链通过共享边缘实现较弱的 结合 ${ }^{[26-27]}$ 。

表 3 为不同 $\mathrm{TiO}_{2}$ 掺杂量下双层中空纤维膜的性 能。如表所示, 随着 $\mathrm{TiO}_{2}$ 掺杂量的增加, 膜的收缩 率小幅增加, 并逐渐接近 $300 \mathrm{~nm}$ 单层纤维收缩率, 表明内层掺杂 $\mathrm{TiO}_{2}$ 有利于提高内层粒子间的熔融 烧结程度。与纯 $\mathrm{Al}_{2} \mathrm{O}_{3}$ 双层膜相比, 掺杂 $\mathrm{TiO}_{2}$ 双层 膜的机械强度明显提升。当 $\mathrm{TiO}_{2}$ 掺杂量为 $2 \mathrm{wt} \%$ 时， 膜的断裂负荷最高达到 $24 \mathrm{~N}$ 。随着 $\mathrm{TiO}_{2}$ 掺杂量的增 加, 膜的断裂负荷逐渐下降。由 XRD 结果可知, $\mathrm{TiO}_{2}$ 与 $\mathrm{Al}_{2} \mathrm{O}_{3}$ 反应生成了脆性的 $\mathrm{Al}_{2} \mathrm{TiO}_{5}$, 且其含量随着 $\mathrm{TiO}_{2}$ 掺杂量的增加而逐渐增加, 导致膜的机械强度 逐渐下降, 这一现象与 Kalita 等 ${ }^{[26]}$ 的研究结果类似, 他们也发现膜的机械强度随着 $\mathrm{Al}_{2} \mathrm{TiO}_{5}$ 含量的增加 而逐渐下降。另一方面, 由于 $\mathrm{TiO}_{2}$ 掺杂量的增加促
进了粒子间的烧结程度, 使内层趋于致密化, 因此 膜的平均孔径、孔隙率和纯水通量都逐渐下降。

\section{3 双层膜外层 $\mathrm{Al}_{2} \mathrm{O}_{3} / \mathrm{PESf}$ 质量比的影响}

双层膜的截留效果取决于外层 $\mathrm{Al}_{2} \mathrm{O}_{3}$ 的有效孔 径, 外层 $\mathrm{Al}_{2} \mathrm{O}_{3}$ 的表面孔径可以通过改变外层纺丝 液中的 $\mathrm{Al}_{2} \mathrm{O}_{3} / \mathrm{PESf}$ 质量比进行调节。对双层膜进行 $\mathrm{SEM}$ 表征, 如图 3 所示, 随着外层 $\mathrm{Al}_{2} \mathrm{O}_{3} / \mathrm{PESf}$ 质量 比的增大, 外表面附近的指状孔长度逐渐变短, 膜 表面的孔径逐渐减小。这是因为当 $\mathrm{Al}_{2} \mathrm{O}_{3} / \mathrm{PESf}$ 质量 比增加时, 外层纺丝液的粘度逐渐增加(如表 4 所示), 此时纺丝液中溶剂与外凝固浴的交换速率降低, 因 此靠近外侧的指状孔长度逐渐缩短。Kingsbury 等 ${ }^{[5]}$ 认为纺丝液粘度的增加抑制了粘性指进现象, 所以 指状孔长度缩短。当纺丝液 $\mathrm{Al}_{2} \mathrm{O}_{3} / \mathrm{PESf}$ 质量比为 13.3 时, 指状孔集中在外层区域, 且孔径很小。此外, 外层 $\mathrm{Al}_{2} \mathrm{O}_{3}$ 密度也随 $\mathrm{Al}_{2} \mathrm{O}_{3} / \mathrm{PESf}$ 质量比的增加而增 大, 经高温烧结后, 聚合物 PESf 完全脱除, 因此外 层趋于致密，表面孔径逐渐减小。

表 4 为外层纺丝液 $\mathrm{Al}_{2} \mathrm{O}_{3} / \mathrm{PESf}$ 质量比对纺丝液 粘度及膜的性能影响, 从表中可以看出由于外层比 内层薄, $\mathrm{Al}_{2} \mathrm{O}_{3} / \mathrm{PESf}$ 质量比的变化对膜整体的断裂 负荷、孔隙率影响很小, 膜的断裂负荷和孔隙率分 别在 $21 \sim 25 \mathrm{~N}$ 和 $49.9 \% \sim 53.91 \%$ 范围内小幅变化。而 膜的纯水通量随 $\mathrm{Al}_{2} \mathrm{O}_{3} / \mathrm{PESf}$ 质量比的增加逐渐下 降。当 $\mathrm{Al}_{2} \mathrm{O}_{3} / \mathrm{PESf}$ 质量比从 2.75 提高至 13.30 时, 纯 水通量从 1.99 降低至 $0.48 \mathrm{~m}^{3} \cdot \mathrm{m}^{-2} \cdot \mathrm{h}^{-1}$, 油截留率从 $97.3 \%$ 小幅提高至 $97.8 \%$ 。由图 3 可知, 这主要是因 为随着外层粒子堆积密度的增加, 外层趋于致密化, 传质阻力逐渐增大, 油截留率略有提高。图 4 为在 不同外层 $\mathrm{Al}_{2} \mathrm{O}_{3} / \mathrm{PESf}$ 质量比条件下双层中空纤维膜

表 $3 \mathrm{TiO}_{2}$ 掺杂量对双层中空纤维膜性能的影响

Table 3 Influence of $\mathrm{TiO}_{2}$ content on properties of dual-layer hollow fiber membranes

\begin{tabular}{cccccc}
\hline $\mathrm{TiO}_{2}$ content $/ \mathrm{wt} \%$ & $\eta / \%$ & Fracture load $/ \mathrm{N}$ & Pure water flux $/\left(\mathrm{m}^{3} \cdot \mathrm{m}^{-2} \cdot \mathrm{h}^{-1}\right)$ & Porosity $/ \%$ & Average pore size $/ \mu \mathrm{m}$ \\
\hline 0 & 14.3 & 12 & 2.30 & 50.89 & 0.240 \\
2 & 14.9 & 24 & 1.65 & 48.05 & 0.152 \\
5 & 14.9 & 22 & 1.32 & 49.97 & 0.147 \\
10 & 15.2 & 20 & 1.21 & 46.43 & 0.150 \\
15 & 15.3 & 19 & 1.12 & 45.68 & 0.145 \\
20 & 16.3 & 17 & 0.91 & 43.90 & 0.141 \\
\hline
\end{tabular}



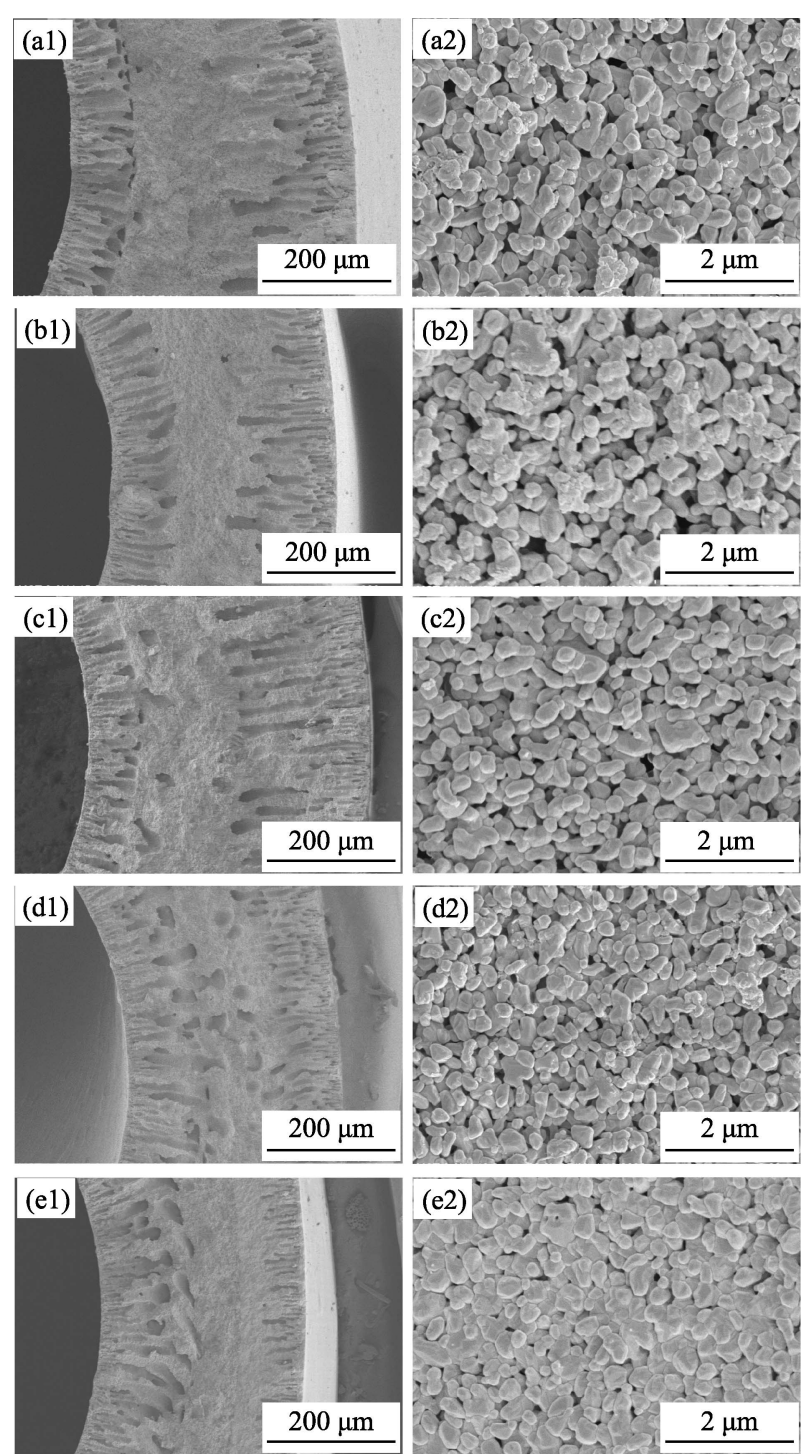

图 3 不同外层纺丝液 $\mathrm{Al}_{2} \mathrm{O}_{3} / \mathrm{PESf}$ 质量比的双层中空纤维膜 SEM 照片

Fig. 3 SEM images of dual-layer hollow fiber membranes with different $\mathrm{Al}_{2} \mathrm{O}_{3} / \mathrm{PESf}$ mass ratios in outer suspension (a) 2.75 ; (b) 4.33 ; (c) 5.60 ; (d) 7.50 ; (e) 13.3

表 4 外层纺丝液 $\mathrm{Al}_{2} \mathrm{O}_{3} / \mathrm{PESf}$ 质量比对双层中空纤维膜性 能的影响

Table 4 Influence of $\mathrm{Al}_{2} \mathrm{O}_{3} / \mathrm{PESf}$ mass ratio in outer suspension on properties of dual-layer hollow fiber membranes

\begin{tabular}{cccccccc}
\hline $\begin{array}{c}\mathrm{Al}_{2} \mathrm{O}_{3} / \\
\mathrm{PESf}\end{array}$ & $\begin{array}{c}\text { Outer } \\
\text { suspension } \\
\text { viscosity/cP }\end{array}$ & $\begin{array}{c}\text { Fracture } \\
\text { load/N }\end{array}$ & $\begin{array}{c}\text { Pure } \\
\text { water flux/ } \\
\left(\mathrm{m}^{3} \cdot \mathrm{m}^{-2} \cdot \mathrm{h}^{-1}\right)\end{array}$ & $\begin{array}{c}\text { Porosity/ } / \\
\%\end{array}$ & $\begin{array}{c}\text { Mean } \\
\text { pore } \\
\text { size/ } \mu \mathrm{m}\end{array}$ & $\begin{array}{c}R_{\mathrm{TOC}} / \\
\%\end{array}$ \\
\hline 2.75 & 7320 & 21 & 1.99 & 49.90 & 0.180 & 97.3 \\
4.33 & 8580 & 22 & 1.10 & 52.26 & 0.175 & 97.5 \\
5.60 & 9720 & 23 & 1.41 & 53.91 & 0.130 & 97.5 \\
7.50 & 11020 & 25 & 1.08 & 53.31 & 0.125 & 97.6 \\
13.30 & 58300 & 23 & 0.48 & 52.54 & 0.115 & 97.8 \\
\hline
\end{tabular}

的小孔孔径分布结果, 从图 4 可以看出, 膜的小孔 孔径为 $0.1 \sim 0.2 \mu \mathrm{m}$, 随着外层 $\mathrm{Al}_{2} \mathrm{O}_{3} / \mathrm{PESf}$ 质量比的
增加, 孔径逐渐减小, 这与通量规律相似。当 $\mathrm{Al}_{2} \mathrm{O}_{3} /$ PESf 质量比为 5.60 时, 孔径分布最窄, 峰强度最高, 说明此时膜的外表面孔径分布最均匀，此时最可几 孔径为 $0.13 \mu \mathrm{m}$ 。

\section{4 烧结温度的影响}

当膜的内层粉体为 $1 \mu \mathrm{m} \mathrm{Al}_{2} \mathrm{O}_{3} / 2 \mathrm{wt} \% \mathrm{TiO}_{2}$, 外 层粉体为 $300 \mathrm{~nm} \mathrm{Al}_{2} \mathrm{O}_{3}$, 且 $\mathrm{Al}_{2} \mathrm{O}_{3} / \mathrm{PESf}$ 质量比为 5.60 时, 考察了烧结温度对双层膜性能的影响, 如 图 5 所示, 随着烧结温度的升高，外表面附近的指

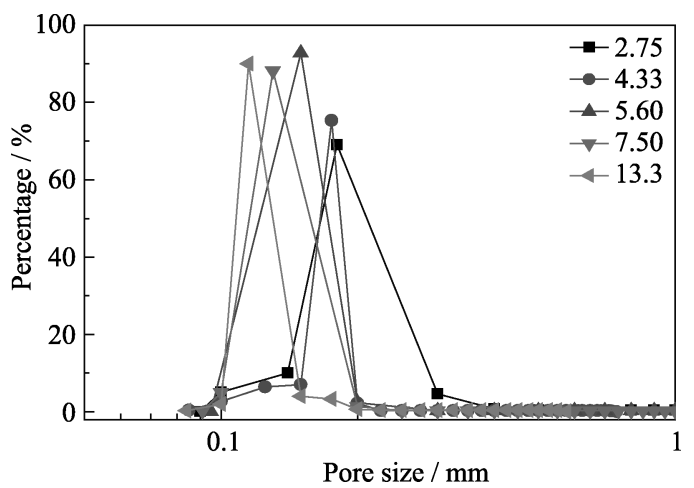

图 4 外层纺丝液 $\mathrm{Al}_{2} \mathrm{O}_{3} / \mathrm{PESf}$ 质量比对双层中空纤维膜孔径 分布的影响

Fig. 4 Influence of $\mathrm{Al}_{2} \mathrm{O}_{3} / \mathrm{PESf}$ mass ratio in outer suspension on pore size distribution of dual-layer hollow fiber membranes
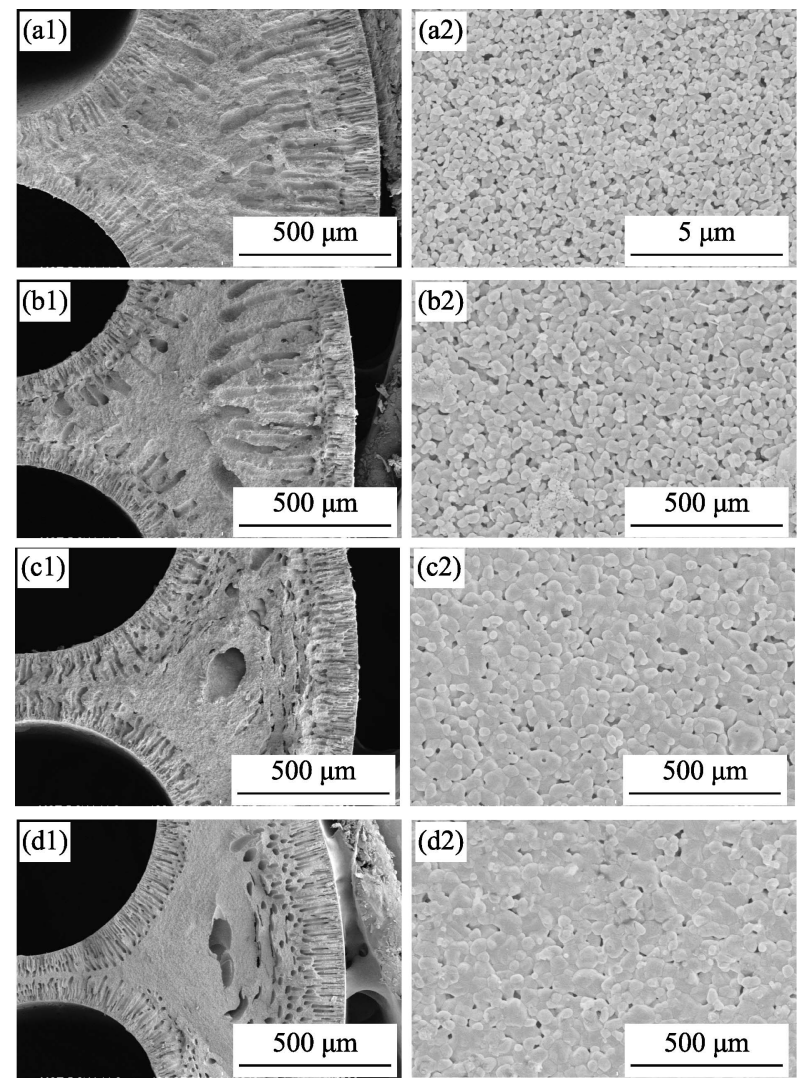

图 5 不同温度烧结的双层氧化铝中空纤维膜的 SEM 照片

Fig. 5 SEM images of dual-layer hollow fiber membrane of alumina sintered at different temperatures

(a) $1250{ }^{\circ} \mathrm{C}$, (b) $1300{ }^{\circ} \mathrm{C}$, (c) $1350{ }^{\circ} \mathrm{C}$, , (d) $1400{ }^{\circ} \mathrm{C}$ 
表 5 不同烧结温度对双层中空纤维性能的影响

Table 5 Influence of sintering temperature on the properties of dual-layer hollow fiber membranes

\begin{tabular}{cccccc}
\hline Temperature $/{ }^{\circ} \mathrm{C}$ & Fracture load $/ \mathrm{N}$ & Pure water flux $/\left(\mathrm{m}^{3} \cdot \mathrm{m}^{-2} \cdot \mathrm{h}^{-1}\right)$ & Porosity $/ \%$ & Average pore size $/ \mu \mathrm{m}$ & $R_{\mathrm{TOC}} / \%$ \\
\hline 1250 & 11 & 3.23 & 61.97 & 0.235 & 96.7 \\
1300 & 15 & 2.34 & 55.62 & 0.199 & 97.4 \\
1350 & 24 & 1.20 & 48.88 & 0.150 & 97.5 \\
1400 & 37 & 0.26 & 40.33 & 0.126 & 98.3 \\
\hline
\end{tabular}

表 6 不同中空纤维陶瓷膜性能比较

Table 6 Comparison in properties of different hollow fiber ceramic membranes

\begin{tabular}{ccccccc}
\hline Membranes & $\begin{array}{c}\text { Sintering } \\
\text { temperature } /{ }^{\circ} \mathrm{C}\end{array}$ & $\begin{array}{c}\text { Average pore } \\
\text { size } / \mu \mathrm{m}\end{array}$ & $\begin{array}{c}\text { Pure water flux/ } \\
\left(\mathrm{m}^{3} \cdot \mathrm{m}^{-2} \cdot \mathrm{h}^{-1}\right)\end{array}$ & $\begin{array}{c}\text { Bending } \\
\text { strength/MPa }\end{array}$ & $\begin{array}{c}\text { Fracture } \\
\text { load/N }\end{array}$ & $\begin{array}{c}\mathrm{Ref} . \\
\text { Single-Channel } \mathrm{Al}_{2} \mathrm{O}_{3} \text { hollow fiber }\end{array}$ \\
Single-Channel $\mathrm{Al}_{2} \mathrm{O}_{3}$ hollow fiber & 1455 & 0.10 & 0.664 & 88.2 & - & {$[28]$} \\
4-Channel $\mathrm{Al}_{2} \mathrm{O}_{3}$ hollow fiber & 1500 & 0.75 & 1.280 & 85.8 & - & {$[29]$} \\
7-Channel $\mathrm{Al}_{2} \mathrm{O}_{3}$ hollow fiber & 1300 & 1.20 & 2.710 & - & 22 & {$[25]$} \\
7-Channel $\mathrm{YSZ} / \mathrm{Al}_{2} \mathrm{O}_{3}$ hollow fiber & 1400 & 230.00 & 1.070 & - & 14 & {$[30]$} \\
4-Channel $1 \mu \mathrm{m} / 300 ~ \mathrm{~nm} \mathrm{Al}_{2} \mathrm{O}_{3}$ hollow fiber & 1350 & 0.15 & 1.260 & - & 24 & This work \\
\hline
\end{tabular}

状孔长度逐渐缩短, 颗粒之间的颈部连结现象逐渐 明显, 膜表面趋于致密。

表 5 为烧结温度对膜的断裂负荷和纯水通量, 以及孔隙率和平均孔径性能的影响。随着烧结温度 的升高, 粒子之间连结更加紧密, 孔隙率逐渐降 低, 平均孔径减小, 纯水通量也随之减小, 而截留 率逐渐升高。当烧结温度从 $1250{ }^{\circ} \mathrm{C}$ 升高至 $1400{ }^{\circ} \mathrm{C}$ 时, 孔隙率从 $61.97 \%$ 下降至 $40.33 \%$, 平均孔径从 $0.235 \mu \mathrm{m}$ 减小至 $0.126 \mu \mathrm{m}$, 纯水通量从 3.23 降低至 $0.26 \mathrm{~m}^{3} \cdot \mathrm{m}^{-2} \cdot \mathrm{h}^{-1}$, 截留率从 $96.7 \%$ 提高到 $98.3 \%$ 。同 时烧结温度的升高有利于提高膜的断裂负荷, 当烧 结温度为 $1400{ }^{\circ} \mathrm{C}$ 时, 膜的断裂负荷高达 $37 \mathrm{~N}$ 。值 得注意的是, 当烧结温度为 $1350{ }^{\circ} \mathrm{C}$ 时, $\mathrm{Al}_{2} \mathrm{O}_{3}-\mathrm{TiO}_{2} /$ $\mathrm{Al}_{2} \mathrm{O}_{3}$ 双层膜的平均孔径为 $0.15 \mu \mathrm{m}$, 纯水通量为 $1.20 \mathrm{~m}^{3} \cdot \mathrm{m}^{-2} \cdot \mathrm{h}^{-1}$; 采用 $300 \mathrm{~nm} \mathrm{Al} \mathrm{O}_{3}$ 制得的单层膜平 均孔径约为 $0.13 \mu \mathrm{m}$ 时, 纯水通量仅为 $0.86 \mathrm{~m}^{3} \cdot \mathrm{m}^{-2} \cdot \mathrm{h}^{-1}$, 表明双层膜在截留率相当的情况下, 纯水通量优势更 加显著, 并且膜的断裂负荷也能满足实际应用要求。

从表 6 可以看出, 本文采用共挤出法制备的双 层中空纤维膜不仅具有较高的渗透通量, 而且具有 较大的断裂负荷, 能够满足实际应用的机械强度要 求。更重要的是, 与其它多通道中空纤维氧化铝膜 相比, 双层膜的平均孔径较小, 具有很好的油水分 离效果。膜的烧结温度也较低, 有利于降低生产能 耗和成本。

\section{3 结论}

采用共挤出法制备了双层中空纤维陶瓷复合膜,
其中内层选用 $1 \mu \mathrm{m}$ 的氧化铝粉体用来降低膜的传 质阻力, 外层选用 $300 \mathrm{~nm}$ 的超细氧化铝粉体用来形 成较小的孔径和光滑的膜表面。通过在内层掺杂 $\mathrm{TiO}_{2}$ 粉体促进粒子烧结, 提高膜的机械强度, 改变 外层纺丝液 $\mathrm{Al}_{2} \mathrm{O}_{3} / \mathrm{PESf}$ 质量比调变膜的有效孔径。 当内层 $\mathrm{TiO}_{2}$ 粉体的掺杂量为 $2 \mathrm{wt} \%$ 时, 膜的断裂负 荷最高达 $24 \mathrm{~N}$ 。当外层 $\mathrm{Al}_{2} \mathrm{O}_{3} / \mathrm{PESf}$ 质量比为 5.60 时, 膜的最可几孔径最小为 $0.13 \mu \mathrm{m}$, 且膜表面光 滑。当烧结温度为 $1350{ }^{\circ} \mathrm{C}$ 时, 双层中空纤维膜的纯 水通量为 $1.20 \mathrm{~m}^{3} \cdot \mathrm{m}^{-2} \cdot \mathrm{h}^{-1}$, 断裂负荷为 $24 \mathrm{~N}$, 表明双 层中空纤维膜能够同时提供较高的通量和机械强 度, 并且双层中空纤维膜的油截留率高达 $97 \%$ 以上， 具有广阔的应用前景。

\section{参考文献:}

[1] WANG S X, TIAN J Y, WANG Q, et al. Low-temperature sintered high-strength $\mathrm{CuO}$ doped ceramic hollow fiber membrane: preparation, characterization and catalytic activity. Journal of Membrane Science, 2019, 570-571: 333-342.

[2] LEE M, WU Z T, WANG R, et al. Micro-structured alumina hollow fibre membranes- potential applications in wastewater treatment. Journal of Membrane Science, 2014, 461: 39-48.

[3] HONG J L, MIN K K, JUNG H P. Decompression stripping of carbon dioxide from rich monoethanolamine through porous hydrophobic modified ceramic hollow fiber membrane contactor. Separation and Purification Technology, 2020, 236: 116304-1-8.

[4] YANG N T, TAN X Y, MA Z F, et al. Fabrication and characterization of $\mathrm{Ce}_{0.8} \mathrm{Sm}_{0.2} \mathrm{O}_{1.9}$ microtubular dual-structured electrolyte membranes for application in solid oxide fuel cell technology. Journal of the American Ceramic Society, 2009, 92(11): 2544-2550.

[5] KINGSBURY B F K, LI K. A morphological study of ceramic hollow fibre membranes. Journal of Membrane Science, 2009, 328(1/2): 134-140. 
[6] AZIZ M H A, OTHMAN M H D, HASHIM N A, et al. Fabrication and characterization of mullite ceramic hollow fiber membrane from natural occurring ball clay. Applied Clay Science, 2019, 177: 51-62.

[7] HUBADILLAH S K, OTHMAN M H D, ISMAIL A F, et al. Fabrication of low cost, green silica based ceramic hollow fibre membrane prepared from waste rice husk for water filtration application. Ceramics International, 2018, 44(9): 10498-10509.

[8] HENNE W, DUNWEG G, SCHMITZ W, et al. Method of Producing Dialyzing Membrane. US, D01F1/08, US04164437.1979.08.14.

[9] XIA Q C, LIU M L, CAO X L, et al. Structure design and applications of dual-layer polymeric membranes. Journal of Membrane Science, 2018, 562: 85-111.

[10] ONG Y K, CHUNG T S. Pushing the limits of high performance dual-layer hollow fiber fabricated via (IPS)-P-2 process in dehydration of ethanol. AIChE Journal, 2013, 59(8): 3006-3018.

[11] LI D F, CHUNG T S, WANG R. Morphological aspects and structure control of dual-layer asymmetric hollow fiber membranes formed by a simultaneous co-extrusion approach. Journal of Membrane Science, 2004, 243(1-2): 155-175.

[12] SETIAWAN L, SHI L, KRANTZ W B, et al. Explorations of delamination and irregular structure in poly(amide-imide)-polyethersulfone dual layer hollow fiber membranes. Journal of Membrane Science, 2012, 423: 73-84.

[13] KHAN I U, OTHMAN M H D, ISMAIL A F, et al. Status and improvement of dual-layer hollow fiber membranes via co-extrusion process for gas separation: a review. Journal of Natural Gas Science and Engineering, 2018, 52: 215-234.

[14] SHI G M, WANG Y, CHUNG T S. Dual-layer PBI/P84 hollow fibers for pervaporation dehydration of acetone. AIChE Journal, 2012, 58(4): 1133-1145.

[15] BONYADI S, CHUNG T S. Flux enhancement in membrane distillation by fabrication of dual layer hydrophilic-hydrophobic hollow fiber membranes. Journal of Membrane Science, 2007, 306(1/2): 134-146

[16] JONG J D, BENES N E, KOOPS G H, et al. Towards single step production of multi-layer inorganic hollow fibers. Journal of Membrane Science, 2004, 239(2): 265-269.

[17] DROUSHIOTIS N, OTHMAN M H D, DORASWAMI U, et al. Novel co-extruded electrolyte-anode hollow fibres for solid oxide fuel cells. Electrochemistry Communications, 2009, 11(9): 1799-1802.

[18] OTHMAN M H D, WU Z T, DROUSHIOTIS N, et al. Single-step fabrication and characterisations of electrolyte/anode dual-layer hollow fibres for micro-tubular solid oxide fuel cells. Journal of Membrane Science, 2010, 351(1/2): 196-204.

[19] JAMIL S M, OTHMAN M H D, RAHMAN M A, et al. Properties and performance evaluation of dual-layer ceramic hollow fiber with modified electrolyte for MT-SOFC. Renewable Energy, 2019, 134: 1423-1433.

[20] GONG X, MENG X X, YANG N T, et al. Electrolyte thickness control and its effect on YSZ/Ni-YSZ dual-layer hollow fibres. Journal of Inorganic Materials, 2013, 28(10): 1108-1114.

[21] WU Z T, WANG B, LI K. A novel dual-layer ceramic hollow fibre membrane reactor for methane conversion. Journal of Membrane Science, 2010, 352(1-2): 63-70.

[22] CHENG H D, WANG X B, MENG X, et al. Dual-layer $\mathrm{BaCe}_{0.8} \mathrm{Y}_{0.2} \mathrm{O}_{3-\delta}-\mathrm{Ce}_{0.8} \mathrm{Y}_{0.2} \mathrm{O}_{2-\delta} / \mathrm{BaCe}_{0.8} \mathrm{Y}_{0.2} \mathrm{O}_{3-\delta}$-Ni hollow fiber membranes for $\mathrm{H}_{2}$ separation. Journal of Membrane Science, 2020, 601: 117801-1-9.

[23] CHONG J Y, WANG B, LI K. High performance stainless steelceramic composite hollow fibres for microfiltration. Journal of Membrane Science, 2017, 541: 425-433.

[24] SHI Z Z, ZHANG Y T, CAI C, et al. Preparation and characterization of $\alpha-\mathrm{Al}_{2} \mathrm{O}_{3}$ hollow fiber membranes with four-channel configuration. Ceramics International, 2015, 41(1): 1333-1339.

[25] CAI C, ZHANG Y T, ZHANG C, et al. Microstructure modulation of $\alpha-\mathrm{Al}_{2} \mathrm{O}_{3}$ hollow fiber membranes with four-channel geometric configuration. Asia-Pacific Journal of Chemical Engineering, 2016, 11(6): 949-957.

[26] KALITA S J, SOMANI V. $\mathrm{Al}_{2} \mathrm{TiO}_{5}-\mathrm{Al}_{2} \mathrm{O}_{3}-\mathrm{TiO}_{2}$ nanocomposite: Structure, mechanical property and bioactivity studies. Materials Research Bulletin, 2010, 45(12): 1803-1810.

[27] STANCIU L, GROZA J R, STOICA L, et al. Influence of powder precursors on reaction sintering of $\mathrm{Al}_{2} \mathrm{TiO}_{5}$. Scripta Materialia, 2004, 50(9): 1259-1262.

[28] LEE M, WU Z, WANG R, et al. Micro-structured alumina hollow fibre membranes-potential applications in wastewater treatment. Journal of Membrane Science, 2014, 461: 39-48.

[29] ZHU L, JI J, WANG S, et al. Removal of $\mathrm{Pb}$ (II) from wastewater using $\mathrm{Al}_{2} \mathrm{O}_{3}$-NaA zeolite composite hollow fiber membranes synthesized from solid waste coal fly ash. Chemosphere, 2018, 206: 278-284.

[30] LEE M, WU Z, WANG B, et al. Micro-structured alumina multichannel capillary tubes and monoliths. Journal of Membrane Science, 2015, 489: 64-72.

[31] WANG B, LEE M, LI K. YSZ-reinforced alumina multi-channel capillary membranes for micro-filtration. Membranes, 2016, 6: 5. 